EPJ Web of Conferences 66, 02115 (2014)

DOI: 10.1051/epjconf/20146602115

(C) Owned by the authors, published by EDP Sciences, 2014

\title{
Triaxial strongly deformed structures in the even-even Hf nuclei
}

\author{
Wenchao $\mathrm{Ma}^{1, \mathrm{a}}$ \\ ${ }^{1}$ Department of Physics \& Astronomy, Mississippi State University, Mississippi State, MS 39762, U. S. A.
}

\begin{abstract}
Two rotational bands of distinct character have been identified in ${ }^{164} \mathrm{Hf}$ from a recent experiment at Gammasphere. They are suggested to correspond to the longanticipated triaxial strongly deformed (TSD) bands predicted by theoretical studies. The bands are substantially stronger in intensity and are located at lower spins than the previously observed TSD bands in ${ }^{168} \mathrm{Hf}$, and have been linked to the known states, hereby making ${ }^{164} \mathrm{Hf}$ the best even-even system for the study of TSD structures in the $A \sim 160$ mass region. Cranking calculations based on the modified-oscillator model suggest that the bands are associated with four-quasiparticle configurations involving high- $j$ intruder $\left(i_{13 / 2}\right)^{2}$ proton orbitals. Wobbling mode has not been observed in ${ }^{164} \mathrm{Hf}$ and the possible reasons are discussed.
\end{abstract}

Collective motion associated with triaxial deformed nuclei has been a subject of much interests. Over the past decade, such type of motion has been identified in several mass regions, e.g., the chiral doublet bands in nuclei around ${ }^{133} \mathrm{Ce}[1]$ and ${ }^{135} \mathrm{Nd}$ [2], the chiral bands and $\gamma$-vibrational bands in nuclei near the neutron-rich ${ }^{110,112} \mathrm{Ru}$ [3], and the low-energy $\gamma$-vibrational bands in ${ }^{76} \mathrm{Ge}$ [4], etc. In the $A \sim 160$ region, families of rotational bands based on the wobbling excitations have been identified in the odd- $Z$, even- $N{ }^{161,163,165,167} \mathrm{Lu}$ [5-9] and ${ }_{73}^{167} \mathrm{Ta}_{94}$ [10]. Triaxial strongly-deformed (TSD) bands based on quasiparticle excitations have also been observed in neighboring nuclei, such as ${ }^{164} \mathrm{Lu}$ [11] and ${ }_{69}^{163} \mathrm{Tm}_{94}$ [12]. These findings have been investigated extensively with different theoretical approaches - see, e.g., Refs. [12-16]. In some of the early theoretical studies, Bengtsson and coworkers suggested that TSD minima associated with deformation parameters $\left(\varepsilon_{2}, \gamma\right) \sim\left(0.4, \pm 20^{\circ}\right)$ in the total energy surfaces (PES) are stabilized by large single-particle shell gaps present at proton numbers $Z=71$ and 72 and neutron numbers $N=94$ and 97 [17, 18]. The calculations were based on a cranked, modified-oscillator model [19]. However, it has proved to be a considerable challenge to predict the behavior of TSD bands in specific nuclei, even those differing by only a single nucleon from the well-studied cases above.

While the observed TSD bands in the $Z=71 \mathrm{Lu}$ isotopes and $N=94$ isotones are consistent with the calculated TSD shell gaps, experimental searches in both ${ }_{72}^{166} \mathrm{Hf}_{94}$ [20] and ${ }^{169} \mathrm{Hf}_{97}$ [21] failed to uncover any TSD structure. Thus far, only two weak TSD bands were reported in ${ }^{168} \mathrm{Hf}_{96}$ [22, 23], however, neither structure appears to result from the wobbling mode. Thus, a wobbling excitation has yet to be observed in an even-even nucleus even though Bohr and Mottelson first discussed wobbling for such a system [24]. The presence of a significant $Z=72$ TSD shell gap also seemed questionable. The quasiparticle excitations in the ${ }^{168} \mathrm{Hf}$ TSD bands are very different from those associated with the wobbling bands of the odd-A Lu and Ta nuclei. In a comprehensive study of TSD structures

\footnotetext{
a e-mail: mawc@ra.msstate.edu
} 

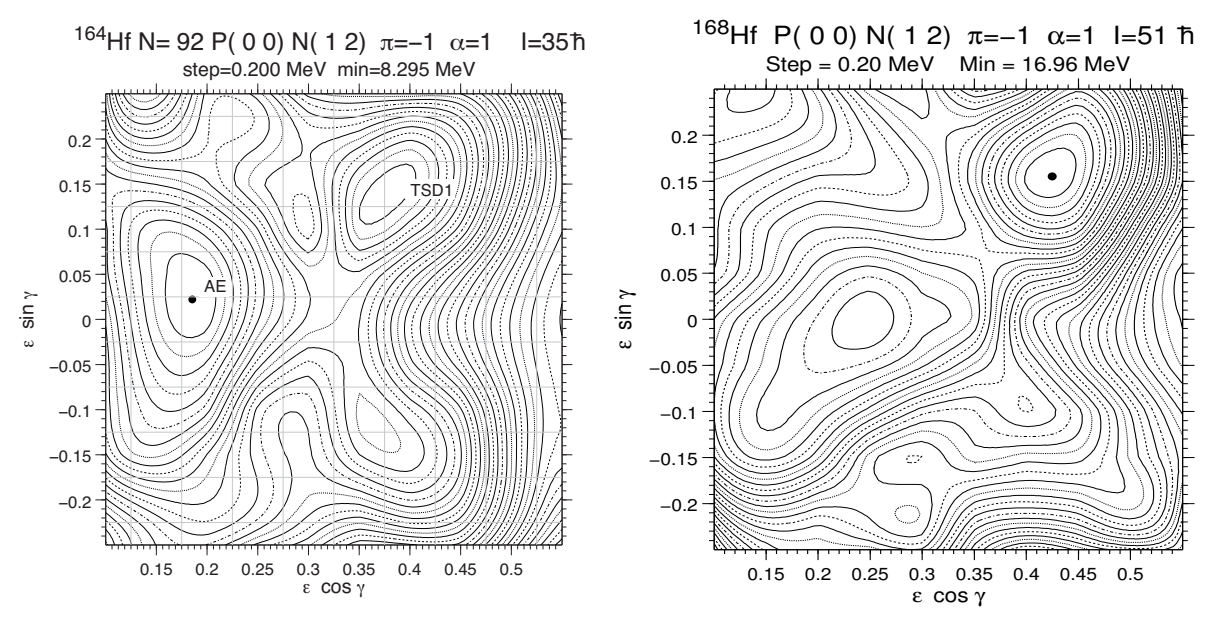

Figure 1. Calculated total energy surfaces for the configuration $(\pi, \alpha)=(-, 1)$ at spin $35 \hbar$ in ${ }^{164} \mathrm{Hf}$ and $51 \hbar$ in ${ }^{168} \mathrm{Hf}$. The energy difference between the contour lines is $0.2 \mathrm{MeV}$.

in the $A \sim 160$ region [25], Bengtsson indicated that the yrast lines of the normal deformed (ND) $N=92$ isotones in this region are systematically higher in excitation energy at high spin than those of their neighbors. This may result in lower relative excitation energies for the TSD bands leading to increased population in fusion-evaporation residues. Thus, ${ }^{164} \mathrm{Hf}_{92}$ could well be a better candidate to study TSD structures than ${ }^{166} \mathrm{Hf}$. The calculated TSD minimum in the TES appears around spins as low as $30 \hbar$ for ${ }^{164} \mathrm{Hf}$, as illustrated in Fig. 1.

Motivated by these considerations, an experimental study of ${ }^{164} \mathrm{Hf}$ was undertaken at the Gammasphere. Two bands with distinctive characters have been revealed and linked to the known normal deformed (ND) states, extending up to spin $48 \hbar$ [26]. The aligned angular momenta of the bands are presented in Fig. 2. The ground-state band GAB undergoes the lowest $i_{13 / 2}$ neutron band crossing (AB crossing) around $\hbar \omega \sim 260 \mathrm{keV}$. The initial alignments of the TSD bands, $\sim 21 \hbar$, are much larger than the alignment of $\sim 11 \hbar$ for the ND band. Furthermore, the TSD bands do not exhibit the proton alignments seen in the ND band at $\hbar \omega \sim 500 \mathrm{keV}$, indicating that the high- $j$ intruder proton orbitals are already occupied at lower rotational frequency in the TSD sequences. Therefore, bands TSD1 and TSD2 are not associated with the ND structures. In addition, the average $J^{(2)}$ values of the TSD bands are more than $30 \%$ larger than that of the ND band AF, as illustrated in Fig. 2. The $J^{(2)}$ values are close to those of the TSD bands in ${ }^{168} \mathrm{Hf}$, indicating that all these TSD bands have similar large deformations, where the measured value of quadrupole moment is $Q_{t} \approx 11.4 \mathrm{eb}$ in ${ }^{168} \mathrm{Hf}$ [22]. Based on these properties, bands TSD1 and TSD2 are proposed to be the long-anticipated TSD bands in ${ }^{164} \mathrm{Hf}$. The bands cross the yrast line at spin $32 \hbar$ with relative intensities of $\sim 2.9 \%$ and $\sim 1.6 \%$, respectively, as compared to a $\sim 50 \hbar$ crossing and an intensity of $\sim 0.26 \%$ for the band TSD1 in ${ }^{168} \mathrm{Hf}$ (see Fig. 3). All these facts make ${ }^{164} \mathrm{Hf}$ the best even-even system to study TSD structures in this mass region.

Theoretical studies suggest that the TSD bands in ${ }^{168} \mathrm{Hf}$ involve the $\pi\left(i_{13 / 2}\right)^{2}$ and the $v\left(j_{15 / 2}\right)$ high- $j$ intruder orbitals; but the latter is not present in the configuration of the TSD bands in ${ }^{164} \mathrm{Hf}$, because of its lower neutron Fermi surface. This interpretation is consistent with an observation that the aligned angular momentum of the TSD bands in ${ }^{164} \mathrm{Hf}$ is $\sim 9.8 \hbar$ larger than that of the ground-state band GAB, see Fig. 2. The corresponding quantity in ${ }^{168} \mathrm{Hf}$ is $\sim 15.5 \hbar$. The difference of $\sim 5.7 \hbar$ is mainly caused by the large aligned angular momentum of the $j_{15 / 2}$ neutron orbital involved in ${ }^{168} \mathrm{Hf}$. It can 

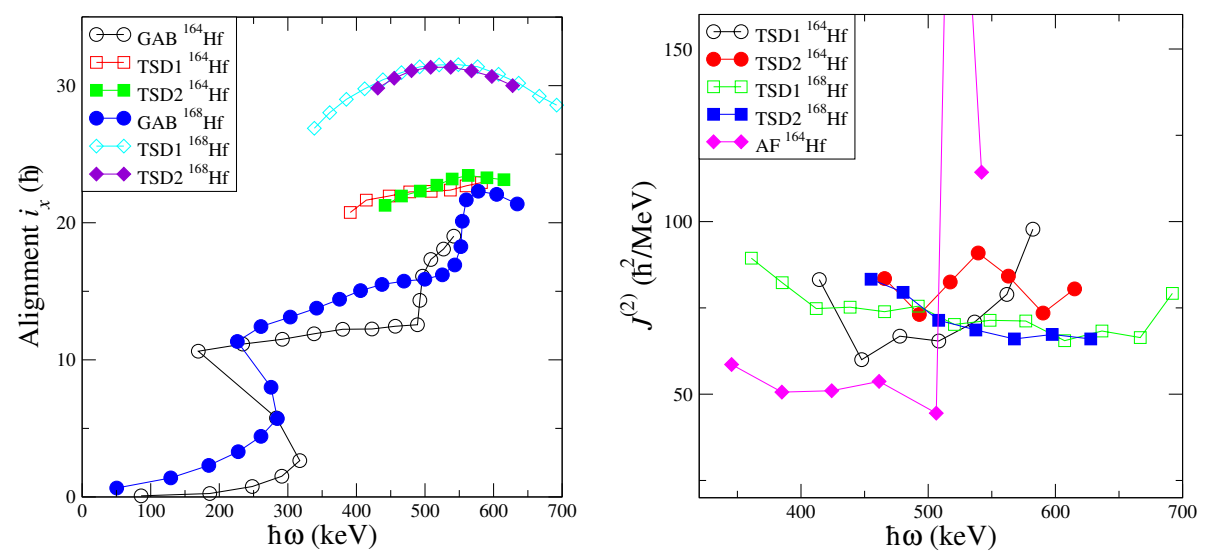

Figure 2. (Color online) Left: Aligned angular momenta $i_{x}$, as a function of rotational frequency $\hbar \omega$, for the ground-state bands and TSD bands in ${ }^{164} \mathrm{Hf}$ and ${ }^{168} \mathrm{Hf}$. Harris parameters $J_{0}=15 \hbar^{2} / \mathrm{MeV}$ and $J_{1}=65 \hbar^{4} / \mathrm{MeV}^{3}$ were used to subtract the angular momentum of the rotating core. The estimated spin values in Ref. [23] are used for TSD bands in ${ }^{168} \mathrm{Hf}$. Right: Dynamical moments of inertia for the TSD bands in ${ }^{164} \mathrm{Hf}$ and ${ }^{168} \mathrm{Hf}$, and for the $\mathrm{ND}$ band $\mathrm{AF}$ in ${ }^{164} \mathrm{Hf}$.

also be seen in Fig. 3 that the slope of TSD bands in ${ }^{164} \mathrm{Hf}$ is smaller than that of TSD bands in ${ }^{168} \mathrm{Hf}$. The value of the slope is closely related to the alignment and the kinematic moment of inertia. The calculations suggest that the difference in the slopes is also caused by the role of the additional high- $j$ intruder orbital, $v j_{15 / 2}$ involved in ${ }^{168} \mathrm{Hf}$. It should be noted that the TSD bands in ${ }^{168} \mathrm{Hf}$ have not yet been linked to the known ND states, and thus, their level spins and energies remain unknown. The ${ }^{168}$ Hf TSD bands are plotted in Figs. 2 and 3 based on the estimated values of spins and energies in Ref. [23], which the readers are referred to for the uncertainties of these quantities.

The wobbling excitation mode, a fingerprint of a stable triaxial nuclear shape, has not been observed in ${ }^{164} \mathrm{Hf}$ and ${ }^{168} \mathrm{Hf}$. Indeed, the intensities of the TSD bands are low, and the observation of collective wobbling excitations based on these bands might be difficult, even if they exist. Such a result may stem from two mechanisms. In the first one, the interaction between wobbling band and surrounding quasiparticle bands leads to a considerable fragmentation of the wobbling amplitude in its wave function, so that it looses to a large degree its unique properties. This mechanism is expected at high excitation energies for wobbling bands. In the second mechanism, the population of the wobbling bands drops below the current observational limit since the total feeding intensity has to be distributed among many excited bands. The situation in odd- $Z$, even- $N$ Lu isotopes, where wobbling excitations were observed, is very different in comparison to the Hf isotopes. The excitation energy of the lowest TSD bands ( $n_{w}=0$ bands) in ${ }^{163,165,167} \mathrm{Lu}$ nuclei is relatively low in comparison to that of other configurations; in particular, it is lower than that of TSD bands based on quasiparticle excitations, see, e.g., Ref. [27] for ${ }^{163} \mathrm{Lu}$. Therefore, it is easier to populate with significant strength wobbling bands built on these $n_{w}=0$ bands, since other configurations are likely located at higher excitation energy and, consequently, the interaction between the wobbling bands and quasiparticle bands is weak, and does not lead to a significant fragmentation of the wobbling amplitude.

Acknowledgments: This work was supported by the U. S. Department of Energy, Office of Nuclear Physics, under grant DE-FG02-95ER40939. 


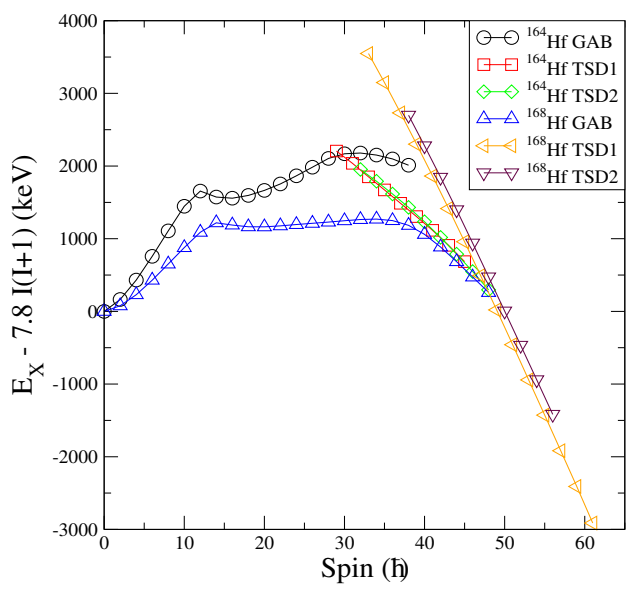

Figure 3. (Color online) Excitation energy, minus a rigid-rotor reference, for the ground-state bands and TSD bands in ${ }^{164} \mathrm{Hf}$ and ${ }^{168} \mathrm{Hf}$. The estimated values of spins and excitation energies in Ref. [23] are used for TSD bands in ${ }^{168} \mathrm{Hf}$.

\section{References}

[1] A. D. Ayangeakaa et al., Phys. Rev. Lett. 110, 172504 (2013).

[2] S. Mukhopadhyay et al., Phys. Rev. Lett. 99, 172501 (2007).

[3] Y. X. Luo et al., Phys. Lett. B 670, 307 (2009).

[4] Y. Toh et al., Phys. Rev. C 87, 041304(R) (2013).

[5] S. Ødegård et al., Phys. Rev. Lett. 86, 5866, (2001).

[6] D. R. Jensen et al., Phys. Rev. Lett. 89, 142503 (2002).

[7] G. Schönwasser et al., Phys. Lett. B 552, 9 (2003).

[8] H. Amro et al., Phys. Lett. B 553, 197 (2003).

[9] P. Bringel et al., Eur. Phys. J. A 24, 167 (2005).

[10] D. J. Hartley et al., Phys. Rev. C 80, 041304(R) (2009).

[11] P. Bringel et al., Phys. Rev. C 75, 044306 (2007).

[12] N. S. Pattabiraman et al., Phys. Lett. B 647, 243 (2007).

[13] I. Hamamoto and G.B. Hagemann, Phys. Rev. C 67, 014319 (2003).

[14] Y. R. Shimizu, T. Shoji, and M. Matsuzaki, Phys. Rev. C 77, 024319 (2008).

[15] B. G. Carlsson, Int'1 J. Mod. Phys, 16, 634 (2007).

[16] K. Sugawara-Tanabe and K. Tanabe, Phys. Rev. C 82, 051303(R) (2010).

[17] H. Schnack-Petersen et al., Nucl. Phys. A 594, 175 (1995).

[18] R. Bengtsson and H. Ryde, Eur. Phys. J. A 22, 355 (2004).

[19] T. Bengtsson, Nucl. Phys. A 496, 56 (1989).

[20] D. R. Jensen et al., Eur. Phys. J. A 8, 165 (2000).

[21] K. A. Schmidt et al., Eur. Phys. J. A 12, 15 (2001).

[22] H. Amro et al., Phys. Lett. B 506, 39 (2001).

[23] R. B. Yadav et al., Phys. Rev. C 78, 044316 (2008).

[24] A. Bohr and B. R. Mottelson, Nuclear Structure, Vol. II, Benjamin, New York, 1975.

[25] R. Bengtsson, [www.matfys.lth.se/staff/Ragnar.Bengtsson/ TSD.html].

[26] J. C. Marsh et al., 2012 Fall Meeting of APS Div. Nucl. Phys., Bull. of APS, Vo. 57, No. 8, 2012; and to be published.

[27] D. R. Jensen et al., Eur. Phys. J. A 19, 167 (2004). 